\title{
ADAM17 silencing suppresses the migration and invasion of non-small cell lung cancer
}

\author{
XIAOHONG LV ${ }^{1}$, YANG LI ${ }^{1}$, MING QIAN ${ }^{2}$, CHENGYUAN MA $^{3}$, \\ HONGYU JING ${ }^{1}$, ZHONGMEI WEN ${ }^{1}$ and DONGHUA QIAN ${ }^{1}$ \\ ${ }^{1}$ Department of Respiration, The First Hospital of Jilin University; ${ }^{2}$ Department of Prosthodontics, Stomatological Hospital, \\ Jilin University; ${ }^{3}$ Department of Neurosurgery, The First Hospital of Jilin University, Changchun, Jilin 130021, P.R. China
}

Received August 24, 2013; Accepted February 24, 2014

DOI: $10.3892 / \mathrm{mmr} .2014 .2029$

\begin{abstract}
A disintegrin and metalloprotease (ADAM) 17 has been implicated in the tumor progression of various types of solid tumor; however, little is known about its role in non-small cell lung carcinoma (NSCLC). The present study evaluated whether the downregulation of ADAM17 affects cell proliferation, the cell cycle, cell migration and cell invasion in NSCLC. A recombinant lentiviral small hairpin RNA (shRNA) expression vector carrying ADAM17 was constructed and then infected into A549 cells, a human NSCLC cell line. Cell proliferation, cell cycle progression, cell migration and cell invasion were determined following the downregulation of ADAM17 by siRNA. It was revealed that downregulation of ADAM17 expression using an RNA silencing approach in A549 tumor cells significantly suppressed cell proliferation and invasion in vitro, and tumor growth in vivo. These data suggested that ADAM17 is an important regulator of the tumorigenic properties of human NSCLC and may be used as a potential anticancer therapeutic target in NSCLC.
\end{abstract}

\section{Introduction}

Lung cancer is the second most common cause of cancer-related mortality worldwide in males and females (1). Non-small cell lung carcinoma (NSCLC), which includes adenocarcinoma, squamous cell carcinoma and large cell carcinoma accounts for $>80 \%$ of lung cancer $(2,3)$. The majority of patients presenting with NSCLC have advanced disease, which precludes curative treatment due to a lack of early diagnosis detection (4). Early detection and treatment may result in the identification of more patients with early central lung cancer and improve survival rates (1). Thus, it is crucial to develop novel molecular

Correspondence to: Dr Donghua Qian, Department of Respiration, The First Hospital of Jilin University, 71 Xinmin Street, Chaoyang, Changchun, Jilin 130021, P.R. China

E-mail: donghuaqian604@163.com

Key words: non-small cell lung cancer, a disintegrin and metalloprotease 17, RNA silencing, proliferation, invasion diagnostic markers and therapeutic targets for the treatment of NSCLC.

A disintegrin and metalloprotease (ADAM) 17 is a member of the disintegrin and metalloprotease gene family. This family encodes proteins that mediate cellular responses to environmental stress by interacting with a variety of cell surface proteins and regulating diverse cellular processes, including proliferation, extracellular matrix binding and ectodomain shedding (5-7). Previously, ADAM17 has been analyzed in various tumor entities and was revealed to be differentially expressed, partially conveying prognostic information $(8,9)$. Previous studies demonstrated that ADAM17 is highly expressed in NSCLC and highly expressed ADAM17 correlates with shortened survival time, suggesting that ADAM17 may also become a useful predictive biomarker for the selection of adjuvant chemotherapy treatment of NSCLC (10). However, little is known about whether the downregulation of ADAM17 effects cell proliferation, cell invasion and the cell cycle in NSCLC, and its underlying molecular mechanisms. Thus, the present study assessed the feasibility of lentiviral vector-delivered small hairpin RNA (shRNA) against ADAM17 for the treatment of NSCLC in vitro and in vivo.

\section{Materials and methods}

Cell culture. The A549 human non-small cell lung cancer cell line was obtained from the Cell Bank of Type Culture Collection of the Chinese Academy of Sciences, Shanghai Institute of Cell Biology (Shanghai, China). A549 lung tumor cells were cultured in Dulbecco's modified Eagle's medium containing $10 \%$ fetal bovine serum (FBS), glutamine (2 mM), penicillin $(100 \mathrm{U} / \mathrm{ml})$, streptomycin $(100 \mathrm{mg} / \mathrm{ml})$ and amphotericin B $(250 \mathrm{mg} / \mathrm{ml}$; all from Invitrogen Life Technologies, Carlsbad, CA, USA) in $5 \% \mathrm{CO}_{2}$ at $37^{\circ} \mathrm{C}$.

Construction and transfection of pGCSIL-GFP-ADAM17. In order to inhibit the expression of ADAM17, an shRNA targeting the ADAM17 transcript was designed. The synthesized oligonucleotides containing a specific target sequence, a loop, the reverse complement of the target sequence, a stop codon for the U6 promoter and two sticky ends were cloned into the pGCSIL-GFP lentivirus vector according to the manufacturer's instructions (Shanghai Gene Chem Co. 
Ltd., Shanghai, China). The target sequence of ADAM17 in the oligonucleotide for suppressing shRNA was: Sense: 5'-GTGCCAGGAGGCGATTAAT-3'. The sequence for the scrambled negative control (NC) for siRNA was: Sense: 5-'AATTCTCCGAACGTGTCACGT-3' . This sequence did not target any gene product and had no significant sequence similarity to human gene sequences, which is essential for determining the effects of siRNA delivery. The lentivirus carrying the ADAM17 siRNA was infected into A549 lung cancer cells as previously described (11).

Quantitative polymerase chain reaction ( $q P C R)$. Total RNA was isolated from A549 cells using TRIzol reagent (Invitrogen Life Technologies) according to the manufacturer's instructions. RNA was reverse-transcribed into cDNA by a PrimeScript ${ }^{\mathrm{TM}}$ RT reagent kit according to the manufacturer's instructions (Takara Bio Inc., Shiga, Japan). qPCR was conducted using the SYBR-Green fluorescent dye method and Rotor Gene 3000 real-time PCR apparatus (Qiagen, Hilden, Germany). The primer sequences were as follows: Forward: 5'-ACTCTGAGGACAGTTAACCAAACC-3' and reverse: 5'-AGTAAAAGGAGCCAATACCACAAG-3' for ADAM17; and forward: 5'-GATCATTGCTCCTCCTGAGC-3' and reverse: 5'-ACTCCTGCTTGCTGATCCAC-3' for $\beta$-actin. The PCR conditions were as follows: A pre-denaturing at $95^{\circ} \mathrm{C}$ for $2 \mathrm{~min}$, followed by 40 cycles of denaturation at $95^{\circ} \mathrm{C}$ for $10 \mathrm{sec}$ and annealing/extension at $55^{\circ} \mathrm{C}$ for $20 \mathrm{sec}$. The amplification specificity was checked by melting curve analysis. The $2^{-\Delta \Delta \mathrm{Ct}}$ method was used to calculate the relative abundance of target gene expression generated by Rotor-Gene Real-Time Analysis Software 6.1.81 (Qiagen). For each cDNA, the target gene mRNA level was normalized to the $\beta$-actin mRNA level.

Western blot analysis. Cultured cells were washed twice with phosphate-buffered saline (PBS), then cells were lysed with Triton X-100 in Hepes buffer [150 mM NaCl, $50 \mathrm{mM}$ Hepes, $1.5 \mathrm{mM} \mathrm{MgCl}_{2}, 1 \%$ Triton $\mathrm{X}-100,0.1 \%$ SDS and a protease inhibitor cocktail (Sigma, St. Louis, MO, USA), $100 \mathrm{mM} \mathrm{NaF}$ and $100 \mathrm{mM} \mathrm{Na}_{3} \mathrm{VO}_{4}$ ] for $30 \mathrm{~min}$. Cell lysates were clarified by centrifugation $(10,000 \mathrm{x} \mathrm{g}$ for $15 \mathrm{~min})$, and protein concentrations were determined using the Bradford reagent (Sigma). The proteins were transferred onto nitrocellulose membranes (Millipore, Bedford, MA, USA) incubated with specific primary antibodies and then the corresponding horseradish peroxidase-conjugated secondary antibodies were added. The specific antibodies used in the western blot analysis were as follows: Antibodies against ADAM17, $\beta$-actin, p21, cyclin D1 and cyclin D3 (Santa Cruz Biotechnology, Inc., Santa Cruz, CA, USA) and matrix metalloproteinase (MMP)-9 and MMP-2 (Sigma). The secondary antibodies used for immunodetection were as follows: Horseradish peroxidase-conjugated goat anti-mouse IgG and goat anti-rabbit IgG (Amersham Biosciences, Uppsala, Sweden). All immunoblots were visualized by enhanced chemiluminescence (Pierce Biotechnology, Inc., Rockford, IL, USA). All the assays using ADAM17 knockdown in A549 cells were performed following the third day of siRNA transfections.

Proliferation assays. To measure the effect of ADAM17 silencing on cell proliferation, the transition of 3-(4,5-dimethylthiazol-2-yl)-2,5-diphenyltetrazolium bromide (MTT) to formazan was used. Cells without any treatment, cells treated only with negative siRNA and cells transfected with siRNAs were seeded in 96 -well plates and kept at $37^{\circ} \mathrm{C}$ for 24,48 and $72 \mathrm{~h}$. The cells were then washed with PBS and incubated in $50 \mu \mathrm{l}$ of $0.5 \mathrm{mg} / \mathrm{ml} \mathrm{MTT}$ in culture medium at $37^{\circ} \mathrm{C}$ for $4 \mathrm{~h}$. Following the addition of $100 \mu \mathrm{l}$ isopropanol, the absorbance was read at $595 \mathrm{~nm}$ by an ELISA plate reader (Molecular Devices Corp., Sunnyvale, CA, USA). The mean proliferation of cells without any treatment was expressed as $100 \%$.

Cell cycle analysis. A549 cells were treated with siRNA for $24 \mathrm{~h}$ in DMEM media containing 5\% FBS. All cells were collected and $1 \times 10^{6}$ cells were centrifuged $700 \times \mathrm{g}$ for $5 \mathrm{~min}$, resuspended in ice-cold $70 \%$ ethanol and stored at $-20^{\circ} \mathrm{C}$ until analysis. Washed cells were stained with $0.1 \%$ Triton X-100 in $0.01 \mathrm{M}$ PBS with $50 \mu \mathrm{g} / \mathrm{ml}$ propidium iodide (Sigma) and $1 \mathrm{mg} / \mathrm{ml} \mathrm{RNase} \mathrm{A}$ (Invitrogen Life Technologies), and incubated at $37^{\circ} \mathrm{C}$ for $30 \mathrm{~min}$ in the dark. The samples of cells were then analyzed for DNA content by FACScan flow cytometry (Beckman Coulter, Inc., Miami, FL, USA) and cell cycle phase distributions was analyzed with the Cell Quest acquisition software (BD Biosciences, Franklin Lakes, NJ, USA). Duplicates were performed in all experiments and experiments were performed on three occasions.

Cell migration assay. The migration assay was performed using a 12-well Boyden Chamber (Neuro Probe, Gaithersburg, MD, USA) with an $8-\mu \mathrm{m}$ pore size. Approximately $1 \times 10^{5}$ cells were seeded into the upper wells of the Boyden Chamber and incubated for $6 \mathrm{~h}$ at $37^{\circ} \mathrm{C}$ in medium containing $1 \% \mathrm{FBS}$. DMEM medium with $10 \%$ FBS was used as a chemoattractant in the bottom wells. Cells that did not migrate through the pores of the Boyden chamber were manually removed with a rubber swab. Cells that migrated to the lower side of the membrane were stained with hematoxylin and eosin and images were captured using an inverted microscope (Olympus, Tokyo, Japan).

Transwell invasion assay. The invasiveness of A549 cancer cells was assessed using 24-well Transwell plates (Corning Inc., Lowell, MA, USA). In brief, $2 \times 10^{5}$ cells in DMEM media with $0.5 \%$ FBS were added to the upper chamber containing $8-\mathrm{mm}$ pore polycarbonate coated with $1 \mathrm{mg} / \mathrm{ml}$ matrigel. The lower chamber was filled with media containing $5 \%$ FBS. Following $16 \mathrm{~h}$ of incubation, the upper surface of the membrane was scrubbed with a cotton-tipped swab. The invading cells on the lower surface of the membrane were fixed and stained with $0.5 \%$ crystal violet. Images of the random fields (five per membrane) were captured at x40 magnification for calculating the cell number. In addition, cells were quantified by measuring the absorbance of dye extracts at $570 \mathrm{~nm}$ in $100 \mathrm{ml}$ Sorenson's solution $(9 \mathrm{mg}$ trisodium citrate, $305 \mathrm{ml}$ distilled water, $195 \mathrm{ml} 0.1 \mathrm{NHCl}$ and $500 \mathrm{ml}$ of $90 \%$ ethanol). Triplicates were performed in all experiments and experiments were performed on five occasions.

Zymographic analysis. MMP-2 and MMP-9 enzymatic activities were measured by gelatin zymography. Conditioned media from cells (cultured without serum for $48 \mathrm{~h}$ ) were collected 
A

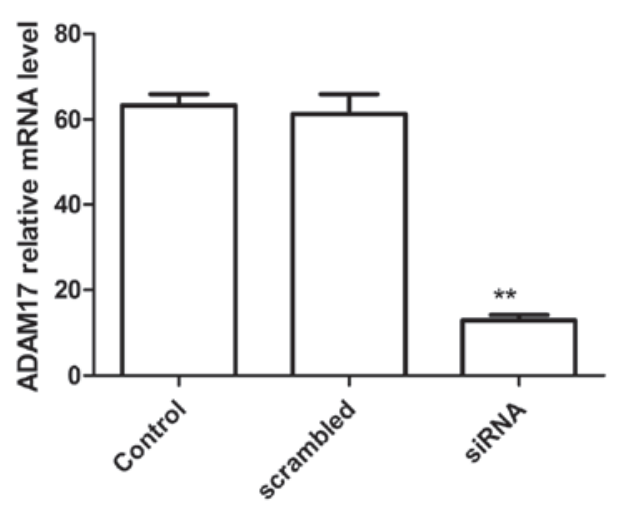

B

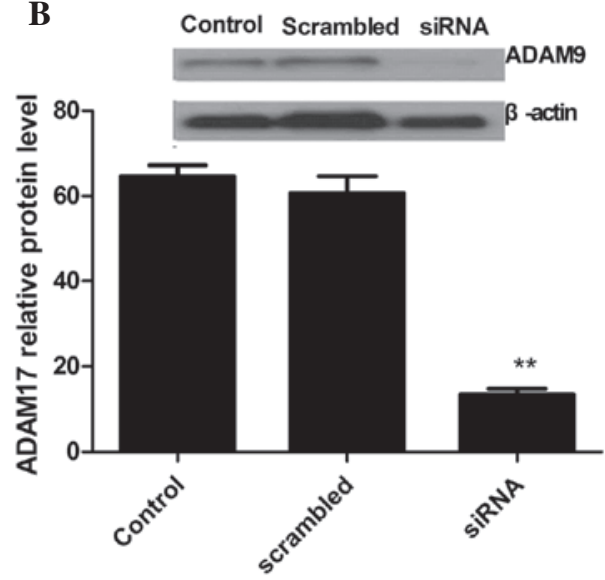

Figure 1. Silencing ADAM17 inhibited the expression of ADAM17 in A549 tumor cells. (A) qPCR analysis of ADAM17 following RNAi silencing; (B) Western blot analysis of ADAM17 following RNAi silencing. ${ }^{*} \mathrm{P}<0.05$ and ${ }^{* *} \mathrm{P}<0.01$ vs. control. ADAM, a disintegrin and metalloprotease; qPCR, quantitative polymerase chain reaction; RNAi, RNA interference; siRNA, small interfering RNA.

and concentrated 20-fold using a Centriprep YM-30 device (Millipore). Samples were then mixed with Laemmli loading buffer and electrophoresed on a gelatin containing $10 \%$ SDS-PAGE. Following electrophoresis, the gel was washed twice with wash buffer (50 mM Tris- $\mathrm{HCl}, \mathrm{pH} 7.5 ; 100 \mathrm{mM}$ $\mathrm{NaCl}$ and $2.5 \%$ Triton $\mathrm{X}-100$ ), followed by a brief rinse in wash buffer without Triton X-100. The gel was then placed in incubation buffer (50 mM Tris- $\mathrm{HCl}, \mathrm{pH} 7.5 ; 150 \mathrm{mM} \mathrm{NaCl}$, $10 \mathrm{mM} \mathrm{CaCl}_{2}, 0.02 \% \mathrm{NaN}_{3}, 1 \mathrm{M} \mathrm{ZnCl}_{2}$ ) at $37^{\circ} \mathrm{C}$ for $24 \mathrm{~h}$. The gel was then stained with Coomassie Blue R-250 and destained with destaining solution. A clear zone of gelatin digestion was representative of the MMP activity.

Tumor xenograft model. All animal experiments were performed in accordance with institutional guidelines, following a protocol approved by the Ethics Committees of the Disease Model Research Center, The First Hospital of Jilin University (Changchun, China). Male, nude mice (age, 6 -weeks) were maintained under specific pathogen-free (SPF) conditions and provided with food and water ad libitum. All the animals were fed with a normal pellet diet one week prior to the experimentation. In vitro cultured A549 cells were injected subcutaneously into mice. The tumor volume was calculated using the following formula: Volume = length $\mathrm{x}$ width ${ }^{2} / 2$. When tumors grew to an average volume of $75 \mathrm{~mm}^{3}$, the mice were randomly divided into the siRNA group, control group (untreated group), NC group ( $\mathrm{n}=10$ in each group) and treated by administration of siRNA or NC plus PBS in a total volume of $20 \mu \mathrm{l}(10 \mu \mathrm{l}$ of the virus plus $10 \mu \mathrm{l}$ of PBS) once a week for 21 days, respectively. When control mice started to succumb to their tumors, mice in all treatment groups were anesthetized by sodium pentobarbital $(1.25 \mathrm{~g} / \mathrm{kg})$ after overnight fasting and decapitated.. The tumors were then removed and directly embedded in an optimal cutting temperature compound in a deep freezer at $-80^{\circ} \mathrm{C}$.

Statistical analysis. Statistical analysis between two samples was performed using Student's t-test. Statistical comparison of more than two groups was performed using one-way analysis of variance followed by Tukey's post hoc test. All data are expressed as the mean \pm standard error of the mean.
The SPSS 13.0 software (SPSS Inc., Chicago, IL, USA) for Windows was used for statistical analyses. $\mathrm{P}<0.05$ or $\mathrm{P}<0.01$ were considered to indicate a statistically significant difference.

\section{Results}

ADAM17 silencing is detected at the mRNA and protein levels. The lentivirus carrying the ADAM17 siRNA or scramble siRNA were transfected into A549 cells. ADAM17 gene expression was markedly decreased in A549 cells treated with siRNA at the mRNA and protein level (Fig. 1) compared with the controls (untreated cell) and scrambled-treated cells. qPCR analysis demonstrated a downregulation of ADAM17 expression in RNAi-mediated knockdown A549 cells when compared with the control cells and scrambled-treated cells (Fig. 1A). At the protein level, similar results were obtained by western blot analysis using an anti-ADAM17 antibody (Fig. 1B).

ADAM17 silencing affects A549 cell proliferation and apoptosis. To investigate whether siRNA affects A549 cell proliferation and apoptosis, A549 cells were treated with siRNA and negative siRNA for 24,48 and $72 \mathrm{~h}$. The antiproliferative and apoptotic effects of siRNA on A549 cells were examined by MTT and acridine orange assays, respectively. It was revealed that ADAM17 silencing was able to significantly inhibit the proliferation of A549 cells when compared with scrambled-treated cells and control cells (Fig. 2A). In addition, to determine the effects of A549 cell cycle progression following ADAM17 silencing, flow cytometry was performed in the present study. The percentage of cells in the siRNA therapy groups significantly increased at the G0/G1 phase compared with scrambled-treated cells and control cells. These results suggest that ADAM17 silencing is able to induce cell cycle arrest at the G0/G1 phase in A549 cells (Fig. 2B).

ADAM17 silencing inhibits A549 cell invasion without affecting cell migration. To analyze whether siRNA effects A549 cell migration, migration assays were performed using Boyden chambers. RNA-mediated ADAM17 silencing 
A

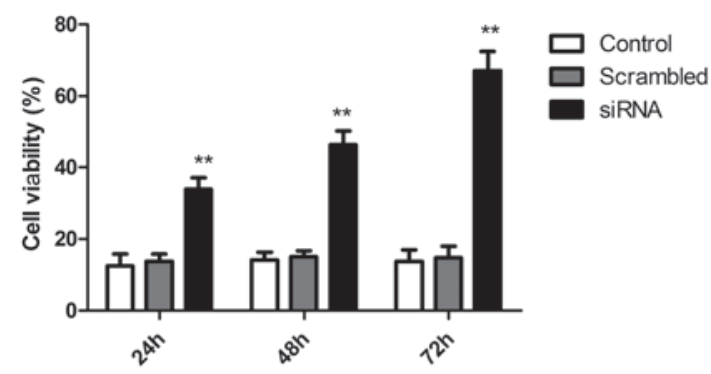

B

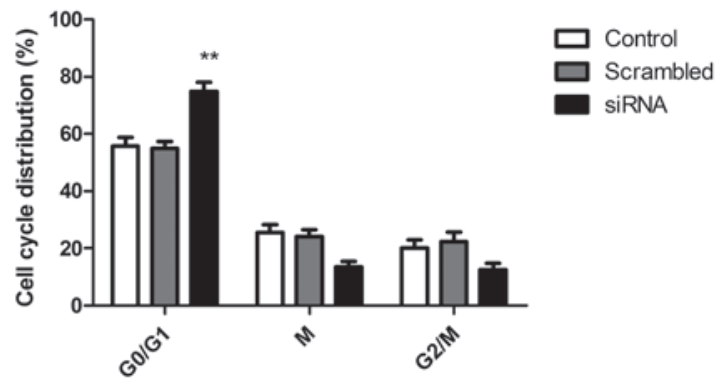

Figure 2. ADAM17 silencing inhibited cell proliferation and induced the cell cycle in vitro. Downregulation of ADAM17 by transfection of siRNA significantly suppressed the proliferation (A) and induced cell cycle arrest (B) in cell cycle arrest at the G0/G1 phase in A549 cells. * P $<0.01$ vs. control. ADAM, a disintegrin and metalloprotease; siRNA, small interfering RNA.

A

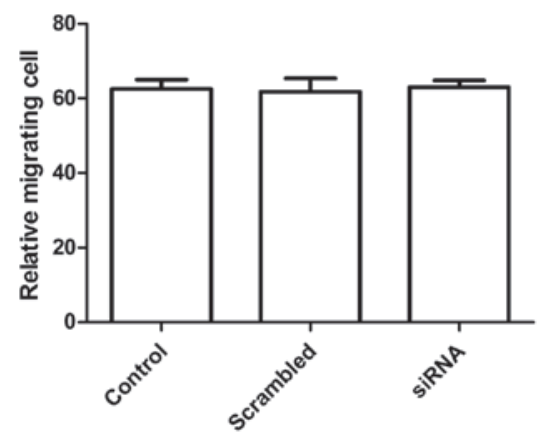

B

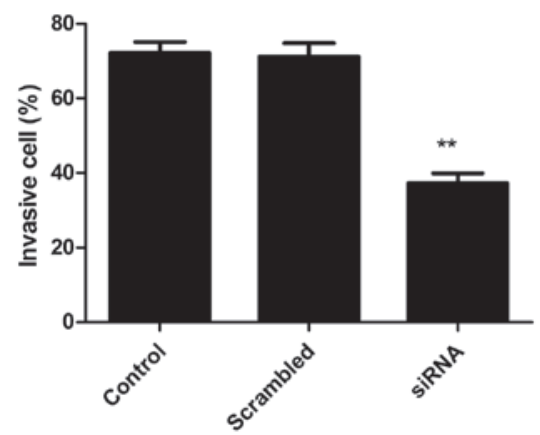

Figure 3. ADAM17 silencing effected cell migration and cell invasion in vitro. Downregulation of ADAM17 by transfection of siRNA (A) had no effect on cell migration and (B) significantly suppressed cell invasion of A549 cells. ${ }^{* *} \mathrm{P}<0.01$ vs. control. ADAM, A disintegrin and metalloprotease; siRNA, small interfering RNA.

had no effect on A549 cell migration when compared with control cells (scrambled-treated cells and untreated cell; Fig. 3A). By contrast, RNAi-mediated ADAM17 knockdown in A549 cells markedly inhibited invasion in vitro when compared with scrambled-treated cells and control cells (Fig. 3B). Control cells (untransfected cell) and NC transfected cells remained invasive and no statistically significant differences were identified.

Silencing ADAM17 suppressed tumor growth in vivo. To investigate the effects of ADAM17 on tumor growth and metastasis in a nude mouse model, a xenograft assay was conducted by injecting the NC cells and ADAM17-silenced tumor cells subcutaneously into mice and comparing the growth rate of the solid tumors. It was revealed that tumor volume and weight was significantly slower for ADAM17 tumor cells compared with the control cells and NC cells following ADAM17 silencing (Fig. 4). These results indicated that the suppression of ADAM17 expression in lung tumor cells markedly suppressed their tumorigenicity in mice.

Preliminary mechanisms involved in the regulation of cell proliferation and invasion by ADAM17. To investigate the preliminary mechanisms involved in the regulation of cell proliferation and invasion by ADAM17 in vitro, the activation of cyclin D1, cyclin D3 and p21 in ADAM17 silencing were analyzed by western blot analysis and compared with that of control cells transfected with empty vectors. As shown in Fig. 5A, ADAM17 silencing significantly inhibited cyclin D1 and cyclin D3 expression and increased p21 expression in the A549 tumor cells.

In order to investigate the mechanisms involved in the inhibition of the invasion ability of RNAi-mediated ADAM17 silencing of A549 cells, zymography assays were performed to evaluate the activity of MMP-2 and MMP-9. Data demonstrated that ADAM17 silencing exhibited much lower MMP-9 activity compared with its control cells as determined by zymography assays (Fig. 5B).

\section{Discussion}

ADAMs are known as ectodomain sheddases, a function of their metalloprotease domains and is a member of the superfamily of $\mathrm{Zn}$-dependent metalloproteinases $(12,13)$. ADAMs are therefore important in the remodeling or processing of cell membrane proteins. Several of the substrates processed by ADAMs, particularly by ADAM10 and ADAM17, have been implicated in the pathogenesis or progression of cancer $(14,15)$. Previous 
A

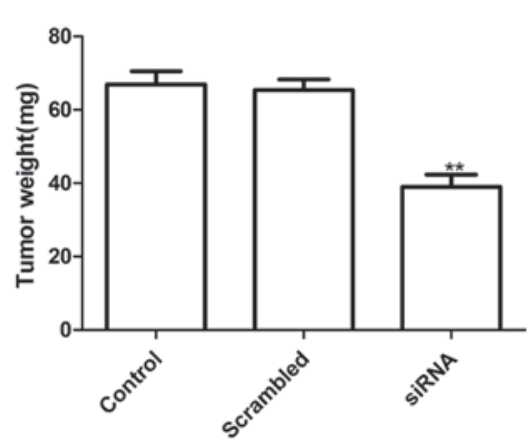

B

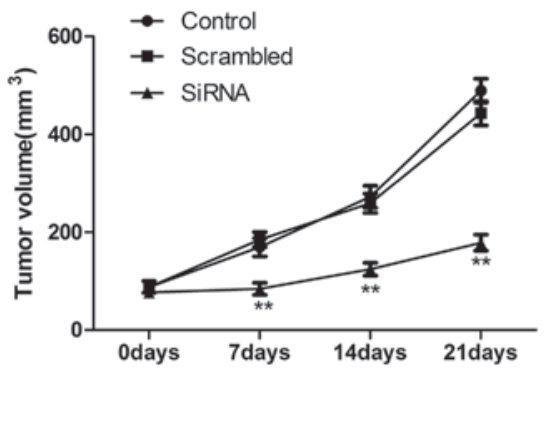

Figure 4. ADAM17 silencing significantly suppressed tumor growth in vivo. (A) Tumor weight in xenograft mice 21 days after silencing ADAM17. (B) Representative images of xenograft tumor models and tumor growth curves of A549 cells. ${ }^{* *} \mathrm{P}<0.01$ vs. control. ADAM, A disintegrin and metalloprotease.

A

\section{Control Scrambled siRNA}

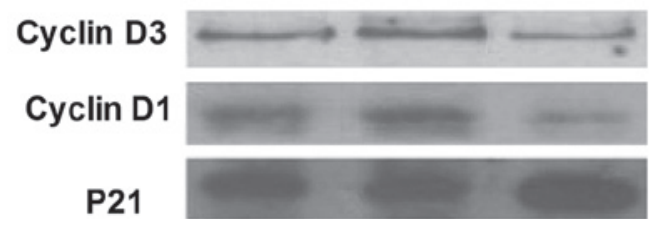

$\mathbf{B}$

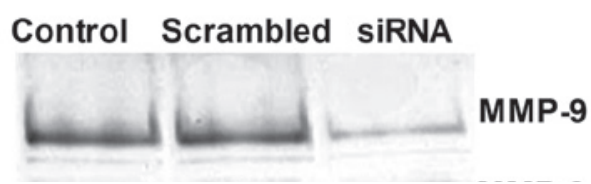

Figure 5. ADAM17 knockdown affects the expression of cell cycle-related protein expression by western blot analysis and MMP activity analysis by gelatin zymography. (A) Protein expression of P21, cylin D1 and cylin D3 was detected by western blotting following ADAM17 knockdown. (B) MMP-2 activity was significantly decreased following silencing ADAM17 when compared with the control cells transfected with scrambled siRNA and untransfected cells, as determined by gelatin zymography. ADAM, a disintegrin and metalloprotease; MMP, matrix metalloproteinase; siRNA, small interfering RNA.

studies have demonstrated that ADAM17 is involved in invasion and proliferation $(16,17)$. The results of the present study are in line with this theory and demonstrate that ADAM17 silencing suppresses A549 tumor proliferation and invasion in vitro and tumor growth in vivo. The data further demonstrated that ADAM17 is important in tumor progression.

ADAM17 sheds a variety of important cell surface molecules, including cytokines and adhesion molecules (18), which are important in cell proliferation and invasion. It has been demonstrated that ADAM17-mediated epidermal growth factor receptor ligand cleavage enhances the proliferation and survival of squamous cell carcinoma cells as well as lung cancer cells $(19,20)$. The present study employed an A549 human lung cancer cell line to investigate the effect of ADAM17 on lung cancer proliferation, cell cycle progression and invasion in vitro. The downregulation of ADAM17 expression was established by siRNA transfection. To assess A549 cell viability and proliferation, an MTT assay was employed. The data demonstrated that a reduction of ADAM17 by shRNA significantly decreased tumor cell proliferation when compared with the control. In addition, the present study also demonstrated that ADAM17 knockdown induces cell cycle arrest at the G0/G1 transition in NSCLC cells. To investigate the exact mechanism, cell cycle-related proteins were analyzed, after silencing ADAM17, by western blot analysis. The p21 protein is a widely accepted cell cycle regulator, as a cyclin dependent kinase (CDK) inhibitor and a negative regulator in the G1/S transition (21). In the present study, compared with the control cells transfected with empty vectors, p21 expression was markedly increased after silencing ADAM17. Whereas, cyclin D1 or cyclin D3 expression decreased following silencing. These data demonstrated that ADAM17 downregulation was able to effect p21, cyclin D3 and cyclin D1 levels to reduce lung carcinoma cell proliferation.

The progression of malignant tumors results from the invasion of the primary tumor to a secondary site, causing metastasis in a multistep process that requires cell-cell and cell-matrix interactions within the host tissue. These interactions lead to the production, release and activation of a variety of cytokines and growth factors and subsequent generation of signals to directly or indirectly promote tumor growth and survival (22). Different proteases have been implicated in these processes, including MMPs, ADAMs and a disintegrin and metalloprotease with thrombospondin motifs $(23,24)$. Due to the strong involvement of ADAM17 in the metastatic process, the present study demonstrated that ADAM17 silencing significantly inhibited the invasion capacity of A549 human lung cancer cells using a matrigel invasion assay, which suggests that this protein is important in cell invasion. Furthermore, it was revealed that ADAM17 silencing was able to inhibit MMP-2 and MMP-9 expression by a zymographic assay indicating reduced cell invasion.

In conclusion, the present study demonstrated that ADAM17 silencing significantly suppressed cell proliferation and invasion in vitro, and tumor growth in vivo. ADAM17 is 
an important regulator of the tumorigenic properties of human NSCLC and may be used as a potential anticancer therapeutic target in NSCLC. Considering the significance of cell invasion in metastatic progression, ADAM17 may be a key target for the design of drugs involved in the treatment or prevention of NSCLC cancer.

\section{References}

1. Siegel R, Naishadham D and Jemal A: Cancer statistics, 2012. CA Cancer J Clin 62: 10-29, 2012

2. Askoxylakis V, Thieke C, Pleger ST, Most P, Tanner J, Lindel K, Katus HA, Debus J and Bischof M: Long-term survival of cancer patients compared to heart failure and stroke: a systematic review. BMC Cancer 10: 105, 2010.

3. Schaake EE, Kappers I, Codrington HE, Valdés Olmos RA, Teertstra HJ, van Pel R, Burgers JA, van Tinteren $H$ and Klomp HM: Tumor response and toxicity of neoadjuvant erlotinib in patients with early-stage non-small-cell lung cancer. J Clin Oncol 30: 2731-2738, 2012.

4. Shepherd FA, Rodrigues Pereira J, Ciuleanu T, et al: Erlotinib in previously treated non-small-cell lung cancer. N Engl J Med 353 123-132, 2005.

5. Fischer OM, Hart S, Gschwind A, Prenzel N and Ullrich A: Oxidative and osmotic stress signaling in tumor cells is mediated by ADAM proteases and heparin-binding epidermal growth factor. Mol Cell Biol 24: 5172-5183, 2004.

6. Mahimkar RM, Visaya O, Pollock AS and Lovett DH: The disintegrin domain of ADAM9: a ligand for multiple betal renal integrins. Biochem J 385: 461-468, 2005.

7. Singh B, Schneider M, Knyazev P and Ullrich A: UV-induced EGFR signal transactivation is dependent on proligand shedding by activated metalloproteases in skin cancer cell lines. Int J Cancer 124: 531-539, 2009.

8. Kenny PA and Bissell MJ: Targeting TACE-dependent EGFR ligand shedding in breast cancer. J Clin Invest 117: 337-345, 2007.

9. McGowan PM, Ryan BM, Hill AD, McDermott E, O'Higgins N and Duffy MJ: ADAM-17 expression in breast cancer correlates with variables of tumor progression. Clin Cancer Res 13: 2335-2343, 2007.

10. Ni SS, Zhang J, Zhao WL, Dong XC and Wang JL: ADAM17 is overexpressed in non-small cell lung cancer and its expression correlates with poor patient survival. Tumour Biol 34: 1813-1818, 2013.

11. Huang WY, Chen DH, Ning L and Wang LW: siRNA mediated silencing of NIN1/RPN12 binding protein 1 homolog inhibits proliferation and growth of breast cancer cells. Asian Pac J Cancer Prev 13: 1823-1827, 2012.
12. Black RA: Tumor necrosis factor-alpha converting enzyme. Int J Biochem Cell Biol 34: 1-5, 2002.

13. Primakoff $P$ and Myles DG: The ADAM gene family: surface proteins with adhesion and protease activity. Trends Genet 16: 83-87, 2000.

14. Duffy MJ, McKiernan E, O'Donovan N and McGowan P: Role of ADAMs in cancer formation and progression. Clin Cancer Res 15: 1140-1144, 2009.

15. Duffy MJ, McKiernan E, O'Donovan N and McGowan P: The role of ADAMs in disease pathophysiology. Clin Chim Acta 403: 31-36, 2009.

16. Takamune Y, Ikebe T, Nagano O and Shinohara M: Involvement of NF-kappaB-mediated maturation of ADAM-17 in the invasion of oral squamous cell carcinoma. Biochem Biophys Res Commun 365: 393-398, 2008.

17. Zheng X, Jiang F, Katakowski M, Zhang ZG, Lu QE and Chopp M: ADAM17 promotes breast cancer cell malignant phenotype through EGFR-PI3K-AKT activation. Cancer Biol Ther 8: 1045-1105, 2009.

18. Ding X, Yang LY, Huang GW, Wang W, Lu WQ: ADAM17 mRNA expression and pathological features of hepatocellular carcinoma. World J Gastroenterol 10: 2735-2739, 2004.

19. Gschwind A, Hart S, Fischer OM and Ullrich A: TACE cleavage of proamphiregulin regulates GPCR-induced proliferation and motility of cancer cells. EMBO J 22: 2411-2421, 2003.

20. Hart S, Fischer OM and Ullrich A: Cannabinoids induce cancer cell proliferation via tumor necrosis factor $\alpha$-converting enzyme (TACE/ADAM17)-mediated transactivation of the epidermal growth factor receptor. Cancer Res 64: 1943-1950, 2004.

21. Niculescu AB III, Chen X, Smeets M, Hengst L, Prives C and Reed SI: Effects of p21(Cip1/Waf1) at both the G1/S and the G2/M cell cycle transitions: $p R b$ is a critical determinant in blocking DNA replication and in preventing endoreduplication. Mol Cell Biol 18: 629-643, 1998.

22. Yu CC, Tsai LL, Wang ML, Yu CH, Lo WL, Chang YC, Chiou GY, Chou MY and Chiou SH: miR145 targets the SOX9/ADAM17 axis to inhibit tumor-initiating cells and IL-6-mediated paracrine effects in head and neck cancer. Cancer Res 73: 3425-3440, 2013.

23. Mochizuki S and Okada Y: ADAMs in cancer cell proliferation and progression. Cancer Sci 98: 621-628, 2007.

24. Rocks N, Paulissen G, El Hour M, Quesada F, Crahay C, Gueders M, Foidart JM, Noel A and Cataldo D: Emerging roles of ADAM and ADAMTS metalloproteinases in cancer. Biochimie 90: 369-379, 2008. 